Canadian Oncology

Nursing Journal

Revue canadienne

de soins infirmiers

en oncologie

Volume 27, Issue 3 • Summer 2017

elSSN: $2368-8076$ 


\title{
Fear of cancer recurrence: A study of the experience of survivors of ovarian cancer
}

\author{
by Jamie Kyriacou, Alexandra Black, Nancy Drummond, Joanne Power, and Christine Maheu
}

\section{ABSTRACT}

Purpose: The purpose of this study is to better understand fear of cancer (FCR) through the experience of ovarian and fallopian tube cancer survivors.

Methods: This study used a descriptive qualitative design. Twelve participants in remission from ovarian or fallopian tube cancer were recruited. Researchers conducted face-to-face, semi-structured interviews and the content, transcribed verbatim, underwent content analysis.

Results: FCR has been identified as a significant concern for women in remission from ovarian cancer. Four themes emerged from the participants' FCR experience: (a) uncertainty surrounding recurrence; (b) varied beliefs and sources of worry; (c) perceived risk of recurrence; (d) management of FCR.

Implications: Survivorship support can be optimized by nurses by screening for FCR, offering psychosocial support for women at risk for FCR, teaching and reinforcing adaptive coping strategies.

\section{INTRODUCTION}

$\mathrm{O}$ varian cancer is the deadliest of the gynecological cancers and has an average two-year recurrence rate of 50-75\% (Ferrell et al., 2005; Ferrell, Smith, Ervin, Itano, \& Melancon, 2003; Cannistra 2004; Karlan, Markman, \& Eifel, 2005). Not surprisingly, given this high recurrence rate, fear of cancer recurrence $(\mathrm{FCR})$ is a common and important concern reported by women diagnosed with ovarian cancer (Ozga, et al., 2015). FCR is defined as "fear that cancer could return or progress in

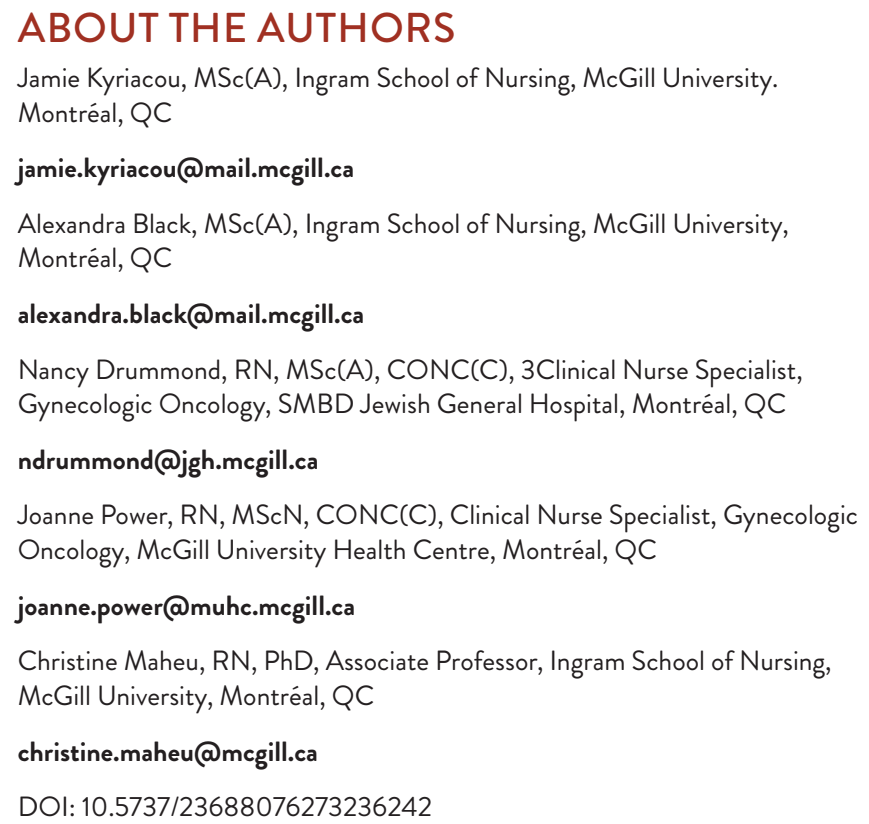

the same place or in another part of the body" (Simard, Savard \& Ivers, 2010; Vickberg, 2003). Unmanaged FCR can lead to anxiety, depression and change in quality of life, all leading to higher emotional, psychological and physical dysfunction (Ozga et al., 2015; Simard et al., 2013). Most studies that previously evaluated the evolution of FCR were conducted in breast cancer patients. To address the significant psychological concern associated with a diagnosis of ovarian cancer, there is a need to better understand the experience of FCR in women diagnosed with this disease (Ozga et al., 2015).

Ovarian cancer is generally diagnosed at an advanced stage due to a lack of a screening tool, resulting in a five-year survival rate of 10-45\% (Surveillance, Epidemiology and End Results Program, n.d.). According to the National Institutes of Health, a survivor is defined as "anyone who has ever been diagnosed with cancer and is living today" (NIH Senior Health, 2015). To date, only one recent systematic review on FCR in ovarian cancer survivor population has been conducted (Ozga et al., 2015). The results of that review (total of fifteen studies, three qualitative and nine quantitative) reported that FCR is an ovarian cancer-specific symptom and its importance is largely a reflection of the high recurrence rate. Due to the various methods of assessing presence of FCR, the reported prevalence range of $22-80 \%$ is broad. Furthermore, FCR levels are similar in participants regardless if they were diagnosed at an early or advanced stage. Presence of FCR was also associated with psychosocial health concerns such as hopelessness, anxiety in the context of death and dying and uncertainty surrounding health status. The poor prognosis for ovarian cancer survivors may explain the paucity of research focusing on the survivorship of these women and, consequently, the examination of their psychosocial needs remains in its infancy (Lockwood-Rayermann, 2006).

Due to the high recurrence rate, the period of remission is brief and is, thus, frequently overlooked as a topic for research, making this the first study to qualitatively explore FCR in ovarian cancer survivors in this timeframe. Specifically, the purpose of this study was to better understand fear of cancer (FCR) through the experience of ovarian and fallopian tube cancer survivors.

\section{METHODS}

\section{Design}

The study used a descriptive qualitative design to obtain a comprehensive description of participants' experiences in their own words (Sandelowski, 2000).

\section{Participants and Recruitment}

Participants were recruited from gynecology-oncology clinics at two university-affiliated hospitals in Montréal, Quebec, using a convenience sampling method. Patients with ovarian, 
primary peritoneal or fallopian tube cancer (collectively referred to as ovarian cancer henceforth given the similarity in characteristics and clinical management of these cancers) who have completed treatment were recruited for this study. The inclusion criteria were that participants: (a) were over 18 years old; (b) spoke English or French; (c) had a previous diagnosis of ovarian, primary peritoneal or fallopian tube cancer; (d) responded "yes" to the question, "Is fear of cancer recurrence a concern for you?"; and (e) in remission. The exclusion criteria were women: (a) with a recurrence of ovarian cancer, primary peritoneal, or fallopian tube cancer; and (b) with a cognitive, neurological or psychiatric impairment that could interfere with successful completion of study procedures, as per the judgment of their Pivot Nurse (a nurse specializing in oncology).

From October to November 2014, 14 women were contacted after having expressed an interest in participating in this study. One woman did not answer follow-up phone calls and another changed her mind about wanting to participate. Twelve women were ultimately interviewed in this study. Interviews were offered in French and English, and all were conducted in English, as per the preference of the participants.

Upon study approval by the institutional review board, potential participants were identified and approached by their Pivot Nurse, a nurse specializing in oncology. Interested participants were contacted by phone by one of two student researchers to explain the study and book the interview.

\section{Data Collection}

Data collection was achieved through one-on-one, faceto-face semi-structured interviews that were conducted in the participant's home or at the hospital. A 13-question interview guide, based on the Model of Fear of Cancer Recurrence (MFCR; Lebel et al., 2014) was used to elicit answers about the women's emotions, thoughts and beliefs about FCR. For example, one question used was, "Can you describe a time when you remember feeling fearful about your risk of recurrence?”

The MFCR (see Figure 1) is an adaptation of Leventhal's Self-Regulation Model of Illness (Leventhal, Diefenbech, \& Leventhal, 1992), refined in 2014 by the work of Lebel and colleagues. It depicts external and internal cues that act as threats during illness and trigger emotions and cognitions, which can both lead to psychological and behavioural responses. The MFCR focuses on the beliefs and thoughts surrounding worry and introduces 'intolerance of uncertainty'. The MFCR also depicts a cyclical relationship between high levels of worrying and an intolerance for uncertainty.

\section{Data Analysis}

The interviews were audio-recorded, transcribed verbatim by the two student researchers. Line-by-line coding was used to break down the data to identify key words and phrases that speak to the research question. Axial coding, the process of organizing codes into categories and linking them with their sub-categories, was then used to condense the codes (Polit \& Beck, 2012). Throughout the analysis, the constant comparative method was used to compare data and codes within transcripts and across transcripts (Loiselle, Profetto-McGrath, Polit, \& Beck, 2010). The process of content analysis was then used to systematically analyze "themes and patterns that emerge in the narrative content" (Polit \& Beck, 2012, p. 505). To ensure interrater consistency, the first four transcripts were each coded separately by the two student researchers, and then compared for similarity in analysis. This process was overseen by the experienced researchers from the research team and based on their suggestions, adjustments were made to ensure consistency.

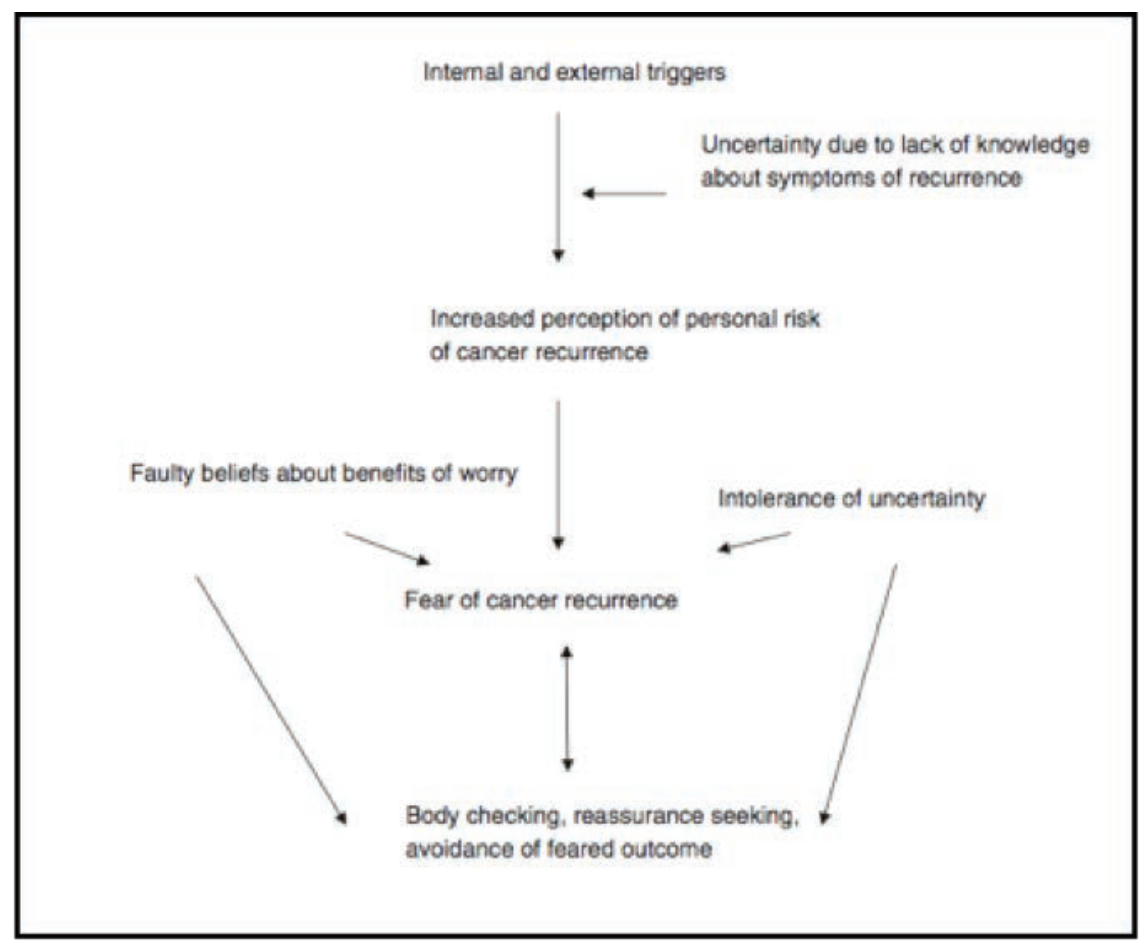

Figure 1. The Model of Fear of Cancer Recurrence 
To establish trustworthiness multiple strategies were employed. Field notes were taken by the two student researchers within 24 hours of having completed the interviews and were used to contextualize the interviews. Peer debriefing, where the two student researchers compared transcripts with each other and with the rest of the research team, was used to improve the interview process (question format and delivery). The same student researcher always contacted the same patients to explain the study, schedule the interview and conduct the interview to achieve consistent and familiar engagement (Guba \& Lincoln, 1994; Polit \& Beck, 2012).

\section{RESULTS}

Twelve women were recruited for this study. Their ages ranged from 37 to 72 years (median $=52$ years) and they were diagnosed with either ovarian cancer $(n=10)$ or fallopian tube cancer $(n=2)$. The time since completion of treatment ranged from 5 to 58 months (median=22 months). Eight of the 12 women had children. Ten women had a family history of cancer and one participant had a previous non-ovarian cancer.

Analysis of the transcripts revealed four major themes: (a) 'feeling certainly uncertain'; (b) 'the many faces of worry'; (c) 'perception of risk of recurrence'; and (d) 'learning to manage'.

\section{Theme 1: Feeling Certainly Uncertain}

Each woman's narrative was etched with the notion of uncertainty, which was a ubiquitous undertone that presented itself in the women's accounts of their FCR. Two facets of uncertainty were revealed by the women: (a) uncertainty over having and recognizing a recurrence; (b) varying tolerance of uncertainty.

Uncertainty in having and recognizing recurrence. Eight out of 12 women questioned whether they could ever know if they were going to have a recurrence or whether they would ever recognize the development of one. "You can't tell, nobody can tell [if cancer is back]", one participant stated. Another challenge that 11 participants faced was recognizing the difference between the vague signs and symptoms of a recurrence and normal bodily symptoms. An example of this is illustrated by one participant, "You know, it could be gas pain and it could be cancer". Furthermore, they perceived that their competence and confidence to determine the source of the signs and symptoms contributed to their uncertainty. "I'm not that confident. I just know, I mean, I know what I should be looking out for," admitted one participant.

Varying tolerance of uncertainty. All the women discussed their differing degree of tolerance for living with the unknown. Nine women perceived themselves as tolerant of uncertainty because they believed that uncertainty is an inevitability of life. For the three women who did identify with intolerance of uncertainty, having precise answers about their prognosis was essential, as conveyed by one of them:

I was all the time... every time I was seeing my doctor, 'Can you give me any percentage? Can you give me any hope?’ And they can't, 'So don't ask me how I am.' 'Don't ask me, 'How are you?' Can you give me any answer? Can you give me any confirmation for later? No? Then don't ask me how I am'.

\section{Theme 2: The Many Faces of Worry}

Worry was a ubiquitous discussion point and affected the women enough to create a panoply of emotions such as stress, anxiety, indifference and a sense of protection. From the narratives, the women attributed part of their fluctuating level of FCR to two facets of worry: (a) the sources of it; (b) their beliefs about it.

Sources of worry. Firstly, worrying about issues indirectly related to cancer emerged from the narratives and those issues seemed to either heighten or supersede the distress associated with FCR. Family was a sensitive topic for four women who worried about the future of their children, grandchildren or parents, should a recurrence ever happen. In these cases, this concern seemed to perpetuate their level of FCR. As one mother stated, "When I look at her [daughter], I'm not allowed to get [pause, holding back tears] because she needs me." Health-related concerns whether normative (i.e., aging) or cancer sequelae (i.e. liver problems, lymphedema, constipation, rheumatoid arthritis, mobility) also preoccupied their thoughts. Since these health issues more acutely affected their activities of daily living, they often superseded their worry about a recurrence and seemingly dimmed their level of FCR. Secondly, in the period leading up to follow-up appointments, all the women reported experiencing lack of sleep, fear, anxiety, physical symptoms, nervousness, intrusive thoughts and stress. These symptoms are all specifically related to the fear that a recurrence would be detected during these follow-ups, as explains one woman, "When I have to go to my appointment, like for the one week before, it's like my nightmare."

Beliefs about worry. When discussing worry for any of the abovementioned issues, three beliefs about worry emerged. Worry can be (a) inevitable; (b) negative; (c) positive. Firstly, three participants admitted that worrying was inevitable, one stating that "you cannot prevent yourself [...] from worrying." Interestingly, despite worry's constant presence, six women insisted that they do not dwell on their worry because it was futile. Secondly, 11 women expressed general negative attitudes towards worry such as robbing them of energy, creating feelings of sadness and leaving them with unanswerable questions. Furthermore, worry was considered by seven women to be detrimental to their physical and/or mental well-being. One woman explained, “... worrying is not good for you, for your mental, for, I don't think even for your body..." Having these negative beliefs about worry seemed to increase the women's FCR. Thirdly, in contrast to the negative attitudes, three women shared their belief that worry could have positive value. One participant described worrying as "a self-defence mechanism", while another explained, "I have to always be on alert" to prevent being surprised if a recurrence happens. For these three women, worrying seemed to decrease the distress associated to their FCR while for the nine other participants, they could not comprehend how worrying could be perceived as beneficial, even to stay vigilant and detect a recurrence earlier.

\section{Theme 3: Perception of Risk of Recurrence}

The third theme revolved around the women's perception of how likely they were to have a recurrence and the impact 
that this perception had on their FCR. An increased perception of risk of recurrence generally led to increased FCR among the participants, although exceptions were noted. Four subthemes were found to affect their personal perception of risk for a recurrence: (a) chemotherapy as insurance; (b) remission milestones and distance from treatment; (c) genetic vulnerabilities to cancer; (d) knowledge of ovarian cancer statistics.

Chemotherapy as insurance. The women's views on the necessity of their chemotherapy influenced their perceived risk of recurrence. Three women believed that their cancer was entirely removed after surgery, and that the chemotherapy "... was like for precaution [pause] just to be on the safe side". Another woman reinforced this idea by saying if she had not had the chemotherapy, and had a recurrence, then she would always wonder, “...maybe I wouldn't be dealing with this.” As a result of this belief, these women experienced a decrease in perception of their risk for a recurrence.

Remission milestones and distance from treatment. Time since completion of treatment and, particularly, the one-, three-, and five-year post-remission milestones were of great significance to the participants. Five women explained their belief that as more time passes their chances for survival improve and, therefore, their perception of risk for a recurrence decreases. As one woman said, "And [MD] said that the longer we go out, the less likely that it's going to come back. So that plays on my mind a lot". The women also hold the remission milestones in high value, giving especial importance to the five-year mark. As one woman explained, "They said that if you're ok for five years, you're cured."

Genetic vulnerabilities to cancer. Four women in this study identified genetic vulnerabilities to cancer, specifically mutations in the BRCA genes, as impacting their risk of recurrence. They believed that positive results may indicate a poorer prognosis and this was distressful for them. One woman expressed her belief, "... especially because I have a mutation [pause] BRCA1. I was tested positive and that means the risk of $85 \%$ of recurrence and maybe breast cancer". Surprisingly, another woman with the same genetic predisposition did not experience higher FCR. For her, however, ovarian cancer was the second primary cancer and, consequently, she no longer feared a recurrence because she had made peace with her increased risk. For two women, testing negative for the mutations, was a great source of relief and, ultimately, decreased their

\begin{tabular}{|c|c|}
\hline \multicolumn{2}{|l|}{ Table 1: Examples of Triggers } \\
\hline Internal & N \\
\hline Thinking of family's future & 4 \\
\hline Appearance of new sign or symptom & 10 \\
\hline External & N \\
\hline Upcoming follow-up appointment & 9 \\
\hline $\begin{array}{l}\text { Decrease in follow-up appointment frequency } \\
\text { Remembering ovarian cancer statistics/own } \\
\text { diagnosis/other people's cancer diagnosis }\end{array}$ & 3 \\
\hline
\end{tabular}

perception of their risk of recurrence and their FCR.

Knowledge of ovarian cancer statistics. Nine participants discussed their knowledge of the survival and recurrence rates of ovarian cancer. Seven women had knowledge of the statistics, which forced them to face the realities of their prognosis, which, in turn, seemed to increase their perception of risk of recurrence. One woman described, "We knew that the success rate or the survival rate was $35 \%$, so a lot of the time I say to myself, 'Well why can't I be that 35\%? Why do I keep thinking that it's going to come back?'” In contrast, for the two women who actively avoided the statistics, their perception of risk seemed decreased.

\section{Theme 4: Learning to Manage}

The women of this study collectively identified over a dozen coping strategies they had developed over time, in response to being continually surrounded by internal or external triggers for their FCR (see Table 1).

As the MFCR depicts, cues trigger FCR and result in certain coping behaviours (see Table 2). When attempting to manage FCR related to a sign or symptom (new or sequela), six women

\begin{tabular}{|l|c|}
\hline \multicolumn{2}{|l|}{ Table 2: Examples of Coping Strategies } \\
\hline & N \\
\hline Body-checking & 6 \\
\hline Systematic checking due to living with sequela(e) & 5 \\
\hline Checking due to appearance of new sign/symptom & 1 \\
\hline Wait and see & 6 \\
\hline Reassurance-seeking & 12 \\
\hline MD & 6 \\
\hline RN & 3 \\
\hline MD and RN & 2 \\
\hline Family/friends & 6 \\
\hline Follow-up appointment & 5 \\
\hline Positive thoughts & 10 \\
\hline Rationalize/normalize/self-talk/consciously move on & 9 \\
\hline Hope & 2 \\
\hline Religion & 2 \\
\hline Self-advocacy & 4 \\
\hline Positive lifestyle & 4 \\
\hline Staying healthy & 2 \\
\hline Living in moment & 1 \\
\hline Helping others & 2 \\
\hline Avoidance & 10 \\
\hline Body-checking & 4 \\
\hline Negative individuals & 3 \\
\hline Sad stories & 5 \\
\hline Ovarian cancer-related issues & 3 \\
\hline
\end{tabular}


engaged in body checking. Four women, however, refused to body check, stating they were scared of what they would find and if it would signal a recurrence. If the body check yielded a red flag, all the women would seek reassurance, but six women would first "wait to see if it goes away." Medical consultation was as equally sought as family and friend support and five women were satisfied with speaking to their Pivot Nurse, feeling that they were "in good hands." Despite it being a common external trigger, the scheduled follow-up was also a source of reassurance for five women, as described by one participant, "Yeah, that follow-up every six months is quite reassuring. I know I will be going to a year... in my brain, I'm going oh no, I don't know about this."

All 12 participants subscribed to one prevailing strategy to manage their FCR, which was to increase the overall "positive" in their life, be it by strengthening positive factors or avoiding negative ones. Ten women altered their way of thinking and learned to either rationalize, normalize or make peace with their situation, or learned to allow themselves to think dark thoughts, but to "not dwell" and to "move on." Two women relied on hope to help decrease their FCR and another two put their fate in "God's hands." Four women felt they became a better self-advocate for their care, which seemed to help them gain control over their FCR. As one woman recalled, "I mean I guess he [gynecology-oncologist] was going to say to come back in a year and I says, 'No! I'm coming back in six months.'” To decrease negativity, 10 participants engaged in avoidance techniques. Four women distanced themselves from individuals who made unhelpful comments or had negative things happening in their life. Along the same lines, three women avoided listening to sad stories of others newly diagnosed with or having died of cancer. Specific to their relationship with ovarian cancer, five women tried not to read about or commit to memory the statistics of recurrence and survival, think about the cancer spreading, recurring or even remember what was removed during surgery. "But most of the time, I live my life as if I didn't have cancer or I'm recovered," is how one participant managed her FCR.

\section{DISCUSSION}

The results of this study offer insight into the experience of FCR, as described by survivors of ovarian cancer. All 12 women of this study discussed living with uncertainty related to the uncertainty about having and recognizing a recurrence. They shared their varying tolerance for living with uncertainty. The women also expressed differing opinions about the value of worrying about a recurrence and identified other sources of worry that could impact their level of FCR. They discussed factors that increased or decreased their perception of risk of having a recurrence. To deal with the challenges they face, the women identified their strategies for managing their FCR.

This study's results regarding uncertainty are consistent with the findings of a literature review of FCR in women with ovarian cancer, which identified uncertainty about having a recurrence as a prominent concern for the women (Ozga et al., 2015). The emergence of worry as a major theme also concurred with the results of a study that found that $80 \%$ of women expressed worry about recurrence at some point (Cesario et al., 2010). Specifically, increased worry in the period leading up to a follow-up appointment was also found in the results of the study by Stewart et al. (2001), which showed that $62.5 \%$ of the 200 women in remission from ovarian cancer reported high to extreme anxiety in that period.

\section{The Paradoxical Medical Follow-up}

While other studies have identified the follow-up appointments as being a source of worry and also a source of reassurance, no study has discussed this contradiction. The women of this study discussed medical follow-up appointments and perceived them as paradoxical-on the one hand triggering FCR and on the other as being reassuring. All 12 women identified feeling apprehension and anxiety leading up to their routine medical follow-ups and it acted as a trigger for their FCR, findings that are consistent with the literature (Lebel et al., 2014; Lewis et al., 2009b; Reb 2007). Furthermore, in congruence with previous studies, participants of this study reported symptoms such as insomnia, irritability, moodiness, tearfulness, depression, and difficulties with concentration and memory (Beaver \& Luker, 2005; Lydon, Beaver, Newbery, \& Wray, 2009; Ozga et al., 2015; Pennery \& Mallet, 2000). From this, the weeks leading up to the follow-up appointments clearly represent a vulnerable time for ovarian cancer survivors.

In contrast to the anxiety leading up to the appointments, many women in this study also expressed feeling a strong sense of reassurance from being followed closely and frequently. This demonstrates the essentialness of medical follow-ups for many of the women and the psychosocial needs of survivors were found to be very high during these follow-up appointments (Lewis et al., 2009a, 2009b; Lydon et al., 2009), providing an opportunity for the healthcare team to support patients. Busy oncology clinics, however, have been said to lack the resources to address these issues, and one solution that has been proposed is that the post-treatment follow-ups be done in conjunction with a specialized nurse (Lewis et al., 2009a, 2009b). These studies have found that specialist oncology nurses are in an optimal position to provide support for the psychosocial and informational needs of the survivors.

\section{Coping with Ovarian Cancer as a Chronic Illness}

The women of this study impressively identified over a dozen strategies to manage their varying levels of FCR. It is important to consider that from 1975 to 2006, the five-year survival rate for all types of ovarian cancer has increased from 33.6\% to 45.2\% (Surveillance, Epidemiology and End Results Program, n.d.). This rise in survivorship is celebration-worthy, but the trade-off is that these women must live with the chronic consequences of cancer (World Health Organization, 2015). Living long-term with FCR is the aftermath of surviving their illness and it is, therefore, primordial that these women cope in a healthy fashion.

Survivorship studies that focus on how to manage ovarian cancer, as a chronic illness, are sparse (Schulman-Green, Nicholson Jr., \& Indeck, 2012). Given the number and nature of coping strategies mentioned in this study, the women are already attempting to self-manage. In simple terms, they 
are trying to effectively become responsible for their daily care over the course of their now chronic disease (Lorig and Holman, 2003). In one qualitative study on health professionals' and participants' perspectives of follow-up treatment of ovarian cancer, the clinicians' narratives revealed that it is feasible for patients to take control of their disease by learning to self-manage, but they must be given satisfactory and timely education, guidance and support (Lydon et al., 2009). One review found that self-management strategies, implemented at various stages of the illness trajectory, minimized the impact of ovarian cancer and its treatment, and were useful in dealing with psychosocial health concerns, including FCR (McCorkle et al., 2009). Ovarian cancer is no longer a death sentence (Reb, 2007), but if FCR is left unaddressed and coping strategies are maladaptive, high-baseline FCR will remain chronically high (Simard et al., 2013).

\section{Strengths and Limitations}

The women of this study were all diagnosed with ovarian cancer, a population that is often overlooked or grouped with other cancers (Lockwood-Rayermann, 2006). This study was also the first to qualitatively explore the experience of FCR in participants who have not yet had a recurrence. Furthermore, this study put in place several measures, including field notes and peer debriefing, to ensure the trustworthiness of the data collected.

This study also has limitations that should be acknowledged. The strength of the relationship between the participant and their Pivot Nurse, as indicated in other literature (Duthie, 2014), could skew the sample towards a subset of survivors who seek out an ongoing therapeutic relationship, making the findings around reassurance-seeking possibly misrepresentative. Additionally, one participant was in remission from both a first primary non-ovarian cancer and a second primary ovarian cancer, but perceived the latter as a recurrence of the former. Her FCR experience, while insightful, was an outlier in the study sample, which leads to the recommendation of adding the exclusion criterion 'having a primary non-ovarian cancer diagnosis' in future studies.

\section{REFERENCES}

Beaver, K., \& Luker, K.A. (2005). Follow-up in breast cancer clinics: Reassuring for patients rather than detecting recurrence. PsychoOncology, 14, 94-101.

Cannistra, S.A. (2004). Cancer of the ovary. New England Journal of Medicine, 351, 2519-2529.

Cesario, S.K., Nelson, L.S., Broxson, A., et al. (2010). Sword of Damocles cutting through the life stages of women with ovarian cancer. Oncology Nursing Forum, 37(5), 609-617.

Duthie, K. (2014). Living with cancer and other chronic conditions: Patient's perceptions of their healthcare experience. Ingram School of Nursing, McGill University.

Ferrell, B.R., Smith, S.L., Ervin, K.S., Itano, J., \& Melancon, C. (2003). A qualitative analysis of social concerns of women with ovarian cancer. Psycho-Oncology, 12, 647-663.

Ferrell, B., Cullinane, C.A., Ervin, K., Melancon, C., Uman, G.C., \& Juarez, G. (2005). Perspectives on the impact of ovarian cancer: Women's views of quality of life. Oncology Nursing Forum, $32,1143-1149$.

\section{Implications for Research and Practice}

Based on the findings of this study, the women fell on a large spectrum with regard to the severity of their FCR. Therefore, for future research on this topic, it would be interesting to explore the relationship between the magnitude of FCR, using a scale such as the Fear of Cancer Recurrence Inventory by Simard and Savard (2009), and the corresponding experiences of the ovarian cancer survivors. While this study did anecdotally note some differences in the experiences of the women with high or low FCR, it was not measured or analyzed.

With respect to nursing practice, the findings of this study may serve as a prompting for nurses working with ovarian cancer survivors to recognize and support women with their FCR. Routine screening of FCR, using validated tools, could prove beneficial in identifying women who are at risk of FCR and its chronic psychosocial sequelae. Furthermore, psychosocial needs are high leading up to and during follow-up appointments, however, medical follow-ups often fall short of addressing these needs. A possible solution would be to also conduct nurse-led follow-ups to address these psychosocial needs and provide more appropriate support for that period of time. Additionally, the vagueness of the signs and symptoms of recurrence was shown to elevate the women's FCR, as they struggled to differentiate between natural body symptoms and recurrence. Verification of the symptoms with their Pivot Nurse helped the participants of this study reduce their FCR. It is, therefore, a recommendation that all patients have access to a similar advanced practice nurse. Finally, in light of ovarian cancer being perceived as a chronic illness, more emphasis on support for FCR is needed. Formal programs, such as the nursing-led six-session intervention currently in clinical trial phase by Maheu and colleagues (2016), have been tested and shown to decrease FCR by helping women reduce their reliance on maladaptive coping strategies and practise alternative adaptive coping strategies (Lebel et al., 2014). Programs such as this should be made available to all cancer survivors experiencing FCR to empower them to properly manage and control their FCR.

Guba, E.G., \& Lincoln, Y.S. (1994). Competing paradigms in qualitative research. Handbook of Qualitative Research, 2, 163-194.

Karlan, B., Markman, M., \& Eifel, P. (2005). Ovarian cancer, fallopian tube carcinoma, peritoneal carcinoma. In V.T. DeVita, S. Hellman, \& S.A. Rosenberg (Eds.), Cancer: Principles and practice of oncology (7th ed., pp. 1364-1397). Philadelphia: Lippincott, Williams \& Wilkins.

Lebel, S., Maheu, C., Lefebvre, M., Secord, S., Courbasson, C., Singh, M., ... Catton, P. (2014). Addressing fear of cancer recurrence among women with cancer: A feasibility and preliminary outcome study. Journal of Cancer Survivorship, 8, 485-496.

Leventhal, H., Diefenbach, M., \& Leventhal, E.A. (1992). Illness cognition: Using common sense to understand treatment adherence and affect cognition interactions. Cognitive Therapy and Research, 16, 143-163.

Lewis, R., Neal, R.D., Williams, N.H., France, B., Wilkinson, C., Hendry, M., ... Weller, D. (2009a). Nurse-led vs. conventional 
physician-led follow-up for patients with cancer: Systematic review. Journal of Advanced Nursing, 65, 706-723.

Lewis, R., Neal, R.D., Hendry, M., France, B., Williams, N.H., Russell, D., ... Wilkinson, C. (2009b). Patients' and healthcare professionals' views of cancer follow-up: Systematic review. British Journal of General Practice, 59, e248-259.

Lockwood-Rayermann, S. (2006). Survivorship issues in ovarian cancer: A review. Oncology Nursing Forum, 33, 553-562.

Loiselle, C.G., Profetto-McGrath, J., Polit, D.F., \& Beck, C.T. (2010). Canadian essentials of nursing research. Philadelphia: Lippincott Williams \& Wilkins.

Lorig, K.R., \& Holman, H.R. (2003). Self-management education: History, definition, outcomes and mechanisms. Annals of Behavioral Medicine, 26, 1-7.

Lydon, A., Beaver, K., Newbery, C., \& Wray, J. (2009). Routine follow-up after treatment for ovarian cancer in the United Kingdom (UK): Patient and health professional views. European Journal of Oncology Nursing, 13, 336-343.

Maheu, C., Lebel, S., Courbasson, C., Lefebvre, M., Singh, M., Bernstein, L.J., ... Sidani, S. (2016). Protocol of a randomized controlled trial of the fear of recurrence therapy (FORT) intervention for women with breast or gynecological cancer. BMC Cancer, 16, 291.

McCorkle, R., Dowd, M., Ercolano, E., Schulman-Green, D., Williams, A., Siefert, M.L., ... Schwartz, P. (2009). Effects of a nursing intervention on quality of life outcomes in post-surgical women with gynecological cancers. Psycho-Oncology, 18, 62-70.

NIH Senior Health. (2015, March). Surviving Cancer: Who is a Cancer Survivor? Retrieved from https://nihseniorhealth.gov/ lifeaftercancer/whoisacancersurvivor/01.html.

Ozga, M., Aghajanian, C., Myers-Virtue, S., McDonnell, G., Jhanwar, S., Hichenberg, S., \& Sulimanoff, I. (2015). A systematic review of ovarian cancer and fear of recurrence. Palliative and Supportive Care, 13, 1771-1780.

Pennery, E., \& Mallet, J. (2000). A preliminary study of patients' perceptions of routine follow-up after treatment for breast cancer. European Journal of Oncology Nursing, 4, 138-145.
Polit, D.F., \& Beck, C.T. (2012). Resource manual for nursing research: Generating and assessing evidence for nursing practice. Philadelphia: Wolters Kluwer Health/Lippincott Williams \& Wilkins.

Reb, A.M. (2007). Transforming the death sentence: Elements of hope in women with advanced ovarian cancer. Oncology Nursing Forum, 34(6), E70-E81.

Sandelowski, M. (2000). Whatever happened to qualitative description? Research in Nursing \& Health, 23, 334-340.

Schulman-Green, D., Nicholson, Jr., N.R., \& Indeck, A. (2012). One step at a time: Self-management and transitions among women with ovarian cancer. Oncology Nursing Forum, 39, 354-360.

Simard, S., \& Savard, J. (2009). Fear of Cancer Recurrence Inventory: Development and initial validation of a multidimensional measure of fear of cancer recurrence. Supportive Care in Cancer, 17, 241-251.

Simard, S., Savard, J., \& Ivers, H. (2010). Fear of cancer recurrence: Specific profiles and nature of intrusive thoughts. Journal of Cancer Survivorship, 4, 361-371.

Simard, S., Thewes, B., Humphris, G., Dixon, M., Hayden, C., Mireskandari, S., \& Ozakinci, G. (2013). Fear of cancer recurrence in adult cancer survivors: A systematic review of quantitative studies. Journal of Cancer Survivorship, 7, 300-322.

Stewart, D.E., Duff, S., Wong, F., Melancon, C. \& Cheung, A. (2001). The views of ovarian cancer survivors on its cause, prevention, and recurrence. Medscape Women's Health, 6(5), 5.

Surveillance, Epidemiology, and End Results Program. (n.d.). SEER stat fact sheets: Ovary cancer. Retrieved from Surveillance, Epidemiology, and End Results Program of the National Cancer Institute: Turning cancer data into discovery. http://seer.cancer.gov/ statfacts/html/ovary.html

Vickberg, S. (2003). The Concerns About Recurrence Scale (CARS): A systematic measure of women's fears about the possibility of breast cancer recurrence. Annals of Behavioral Medicine, 25, 16-24.

World Health Organization. (2015). Noncommunicable diseases. Retrieved from World Health Organization: http://www.who.int/ topics/noncommunicable_diseases/en/ 